\title{
An Analytical Model for Fair Rate Calculation in Resilient Packet Rings
}

\author{
Arash Shokrani, Ioannis Lambadaris, and Jérôme Talim \\ Department of Systems and Computer Engineering, Carleton University, Ottawa, Canada \\ \{shokrani, ioannis, jtalim\}@sce.carleton.ca
}

\begin{abstract}
Resilient Packet Ring (RPR) is a new Medium Access Control (MAC) protocol for high-speed ring networks. It supports spatial reuse and, therefore, maintaining fairness among different nodes is a challenging task in RPR. To ensure fairness among nodes, a fairness algorithm is employed at each RPR node. In case of congestion, the fairness algorithm advertises a fair rate to all upstream nodes contributing to the congestion. In this paper, we develop an analytical model for fair rate calculation in the standard RPR fairness algorithm in the parking lot scenario. We first ignore the link propagation delay and model the system using a nonlinear discrete-time low-pass filter. We, then, consider the link propagation delay and develop a more realistic model. We verify our model by simulation results and analyze the effect of various parameters on the convergence time. Finally, we determine the low-pass filter coefficient to ensure that convergence time of the algorithm is within its minimum range.
\end{abstract}

\section{INTRODUCTION}

The Resilient Packet Ring (RPR) IEEE 802.17 standard is a new Medium Access Control (MAC) protocol for ring networks. It is devised to achieve several objectives including high utilization, fault recovery, and fairness, which are not simultaneously available in the current technologies [1], [2]. Packets are removed from the ring at their destinations and, as a result, RPR can support spatial reuse, i.e. multiple concurrent transmissions over different parts of the ring [3]. However, the spatial reuse may cause congestion and unfairness among nodes in accessing the ring bandwidth. Therefore, a fairness algorithm is required to ensure a fair ring access for all nodes.

The ring access scheme in RPR is based on the Buffer Insertion Ring (BIR) method, in which every node (station) on the ring has an insertion (transit) buffer [4]. The ring traffic transiting a node, may be temporarily stored in the transit buffer. Each node is allowed to add its local traffic (add traffic) to the ring when its transit buffer is empty. In other words, ring traffic has non-preemptive priority over the local traffic. This means that the transit traffic at each node can block the local traffic of that node in accessing the ring. Hence, all nodes should be forced to adjust the insertion rate of their local traffic (add-rate) according to their fair shares.

The objective of the fairness algorithm in RPR is to distribute fairly the available bandwidth on any link among the local traffic of all competing nodes on that link. We define the true fair rate as the rate at which every node should add its local traffic to the ring without starving its downstream nodes. When a node is congested, it calculates a first approximation to the true fair rate. The calculated fair rate is then advertised to the upstream nodes through a control message. As the upstream nodes receive the control message, they adjust their add-rates according to the received fair rate. Note that as the calculated fair rate is an approximation to the true fair rate, the congestion may not be cleared at the congested node. In this case, the congested node calculates another approximation of the fair rate and advertises it to the upstream nodes. This process may repeat until the calculated fair rate converges to the true fair rate. The time needed for the fairness algorithm to converge is commonly referred to as convergence time, which is an important criterion in evaluating performance and efficiency of an RPR fairness algorithm.

In [2] and [5], convergence times of different RPR fairness algorithms are investigated based on the simulation results. However, an analytical model provides a solution to evaluate the performance of different RPR fairness algorithms. This paper presents an accurate analytical model for fair rate calculation in the standard RPR fairness algorithm. This analysis is done for the parking lot scenario depicted in Fig. 1, in which all nodes $1,2, \ldots$, and $n$ have infinite local traffic to send to node 0 . This scenario is one of the traditional and challenging benchmark scenarios in RPR. It is studied in standardization process of RPR, as well as in [5], to compare performance of different RPR fairness algorithms. Using this analytical model, we study the performance of the RPR standard fairness algorithm in terms of stability, and convergence time. We also investigate the impact of various system parameters on the performance of the fairness algorithm.

In Section II the RPR node architecture, the notion of fairness in RPR, and the RPR standard fairness algorithm are described. Section III presents an analytical model of fair rate calculation in RPR. Section IV deals with the numerical results. Finally, Section V concludes this paper.

\section{An Overview of Resilient Packet Ring}

RPR supports three traffic classes: high-priority class A; medium-priority class $\mathrm{B}$; and low-priority class $\mathrm{C}$. The bandwidth is allocated for class A and a subclass of class B.

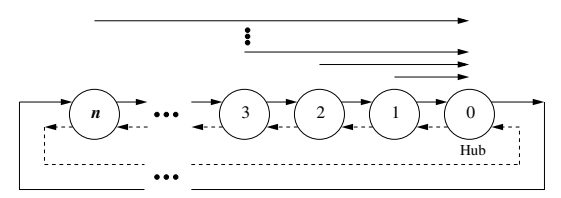

Fig. 1. Parking lot scenario 
The fairness algorithm dynamically divides the unreserved bandwidth among the unallocated portion of class B and class $\mathrm{C}$ traffic of all nodes. The traffic controlled by the fairness algorithm is called fairness eligible (FE).

RPR employs two unidirectional counter-rotating ringlets. Fig. 2 presents an RPR node architecture, where only one direction of the ring traffic is shown. At each node, the arriving traffic from the ring is removed if destined to that node; otherwise, it is forwarded to the transit buffer. When the transit buffer is implemented in single-queue mode, all transiting traffic with different priorities are forwarded into a single queue. In this mode, the scheduler gives the service priority to the transit traffic over the local traffic, which guarantees a lossless ring. The fairness module implements the fairness algorithm, transmits control messages, and adjusts the rate controller of the local traffic.

In RPR, the bandwidth share of each competing node on a given link should be proportional to the relative weight assigned to that node. In other words, a per-station fairness in utilizing the ring bandwidth should be maintained.

\section{A. RPR Standard Fairness Algorithm}

Congestion detection and fair rate calculation in RPR nodes are performed at the end of every fixed time interval called control interval. Each node measures the service rate of its FE local traffic and that of the transit traffic, at the output of the scheduler. The add-rate (respectively, the forward-rate) is the average amount of FE local traffic (respectively, transit traffic) serviced per unit of time; both are measured at the end of each control interval. Let us define the following notations:

- $C$ is the unreserved link capacity called unreserved rate.

- $T$ is the length of the control interval and control interval $k>0$ is the time interval $((k-1) \cdot T, k \cdot T]$.

- $\alpha$ is a low-pass filtering coefficient $(0<\alpha<1)$.

- $r_{i}^{a}(k)$ is the add-rate of node $i$ in control interval $k$ and $\tilde{r}_{i}^{a}(k)$ is its low-pass filtered version defined as follows:

$$
\tilde{r}_{i}^{a}(k)=(1-\alpha) \cdot \tilde{r}_{i}^{a}(k-1)+\alpha \cdot r_{i}^{a}(k) .
$$

- $r_{i}^{f}(k)$ is the forward-rate of node $i$ in control interval $k$ and $\tilde{r}_{i}^{f}(k)$ is its low-pass filtered obtained similar to (1).

- $u_{i}(k)=r_{i}^{a}(k)+r_{i}^{f}(k)$ is the rate of FE traffic at the output link of node $i$ in control interval $k$, and $\tilde{u}_{i}(k)$ is

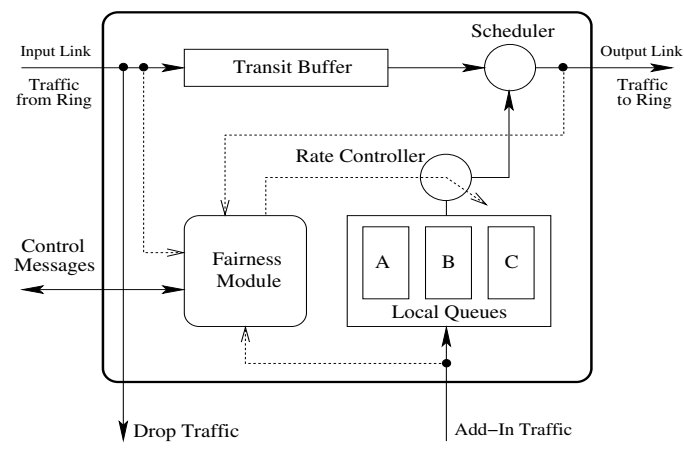

Fig. 2. RPR node architecture its low-pass filtered version. Intuitively, we have

$$
\tilde{u}_{i}(k)=\tilde{r}_{i}^{a}(k)+\tilde{r}_{i}^{f}(k)=(1-\alpha) \cdot \tilde{u}_{i}(k-1)+\alpha \cdot u_{i}(k) .
$$

For simplicity, assume that all rates are normalized to $C$. Therefore, $u_{i}(k) \leq 1$ and $r_{i}^{a}(k) \leq 1-r_{i}^{f}(k)$.

1) Congestion Detection: Assume that node $i$ is implemented in single-queue mode. At the end of control interval $k$, node $i$ detects congestion if $\tilde{u}_{i}(k)$ exceeds a certain threshold $\tau$ (e.g. $0.95 \cdot C$ ); or a packet at the head of the local buffers of that node experiences a long delay to access the ring.

2) Fair Rate Calculation: The RPR standard fairness algorithm operates in two modes: Aggressive Mode (AM) and Conservative Mode (CM) [6]. We assume that all nodes are operating in AM, as this is the default mode of operation in RPR. In AM, when node $i$ detects congestion in control interval $k$, it advertises $\tilde{r}_{i}^{a}(k)$ as the fair rate to its upstream nodes. If node $i$ is not congested, the fair rate is set to 1 .

3) Control Message Transmission: We assume that there is a single control message circulating along the ring within each control interval. It contains separate fields for the calculated fair rate of all nodes. At the end of each control interval, one of the nodes initiates transmission of the control message. This node inserts its advertised fair rate in a designated field and sends the control message to its upstream node. Upon receiving a control message, node $i$ adjusts the rate of its local FE traffic so that its add-rate for destinations beyond node $j$ does not exceed the advertised fair rate of node $j$. It also updates the corresponding field in the control message with its most recently calculated fair rate and forwards the control message to its upstream node.

\section{PROBLEM DESCRIPTION}

We consider the parking lot scenario (Fig. 1) which is a benchmark scenario for evaluating an RPR fairness algorithm. The performance of an RPR fairness algorithm should be evaluated in overloaded conditions; otherwise, congestion does not occur and there is no need for a rate control mechanism. Hence, we assume that nodes $1,2, \ldots$, and $n$ (Fig. 1) have infinite amount of traffic in their local buffers destined to node 0 . Without loss of generality, we only consider FE traffic as the fairness algorithm only controls the rate of FE traffic throughout the ring. Since all nodes compete for the bandwidth on the link between node 1 and node 0 , this link becomes the most congested one. We assume that all nodes have equal weights. Hence, the fair share of each node is $\frac{1}{n}$ of the link capacity. We also assume that the fairness algorithm operates in AM. Note that the transit queue mode is independent of the fairness algorithm and it only specifies how congestion is triggered [6]. We assume that the transit queue at each node is implemented in the single-queue mode. In Fig. 1, the solidline ringlet is the traffic path to node 0 . The control message is transmitted backward on the dashed-line ringlet.

In the following sections, we first assume that the link propagation delay is zero. Then, we incorporate the deterministic link propagation delay into the model and analyze the problem in a more realistic case. 


\section{A. Analysis of the Model without Link Propagation Delay}

In this section we ignore the link propagation delay and model the fair rate calculation in the RPR standard fairness algorithm. Then, we determine the conditions with respect to which the convergence time of the fairness algorithm is finite. Let us define $\ell_{i}(k)$ as the rate limiter value of the local traffic of node $i$ at the beginning of control interval $k$. This is the maximum rate at which node $i$ is allowed to add traffic to the ring destined to node 0 . The add-rate of node $i$ in control interval $k$ is also bounded by $1-r_{i}^{f}(k)$ and hence we have

$$
r_{i}^{a}(k)=\min \left\{1-r_{i}^{f}(k), \ell_{i}(k)\right\} .
$$

1) Congestion Detection Time: Starting at time 0, each node has traffic at link rate to transmit to node 0 . The initial rate limiter value of all nodes is equal to the link rate: $\ell_{i}(1)=$ $1, i=1,2, \ldots, n$. Recall that at each node the transit traffic has priority over local traffic of that node. As a result, before the congestion detection, only node $n$ which is the most upstream node can transmit at the link rate, i.e. $r_{n}^{a}(k)=1$; other nodes will not be able to add their local traffic to the ring before the congestion detection. Hence, $r_{i}^{a}(k)=0, i=1, \ldots, n-1$. Note that as node $n$ sends traffic at the link rate to node 0 , before the congestion detection we have $u_{i}(k)=1, i=1, \ldots, n$.

We assume that the congestion is triggered at node $i$ only when $\tilde{u}_{i}(k)$ exceeds $\tau$. As congestion is detected through a low-pass filtering approach, all nodes detect congestion after several control intervals. For node 1, in particular, we have $\tilde{u}_{1}(0)=0$ and $u_{1}(k)=1$, for all $k$ prior to the congestion detection. Therefore, one can derive $\tilde{u}_{1}(k)$, using (2), as follows:

$$
\tilde{u}_{1}(k)=1-(1-\alpha)^{k} .
$$

Node 1 detects the congestion at the end of control interval $\hat{k}$, where $\hat{k}=\min \left\{k: \tilde{u}_{1}(\hat{k})>\tau\right\}$. Using (4) we obtain

$$
\hat{k}=\left\lfloor\frac{\ln (1-\tau)}{\ln (1-\alpha)}+1\right\rfloor,
$$

where $\lfloor\cdot\rfloor$ is the floor function. Note that nodes $2,3, \ldots$, and $n$, detect the congestion at the same time as node 1 .

2) Advertised Fair Rate: Assume that node 1 initiates the control message transmission at the end of each control interval. As the size of the control message in RPR is relatively short, we can ignore its transmission and processing time. We have also assumed that the link propagation delay is zero in this ideal model. As a result, the time needed for a control message to reach the furthest upstream node in the ring (node $n$ ) is zero. This means that all nodes receive and apply the fair rate with no delay after it is advertised. For $k \leq \hat{k}$, only node $n$ can add traffic to the ring. Therefore, we have

$$
\tilde{r}_{i}^{a}(\hat{k})=0, \quad i=1,2, \cdots, n-1 .
$$

When the congestion is detected, node 1 advertises its calculated fair rate, i.e. $\tilde{r}_{1}^{a}(\hat{k})=0$, to its upstream nodes. Node $i>1$ receives the fair rate instantly and sets its rate limiter to the received fair rate, i.e. $\ell_{i}(\hat{k}+1)=\tilde{r}_{1}^{a}(\hat{k})=0$. Note that the rate limiter value of a node only changes when that node

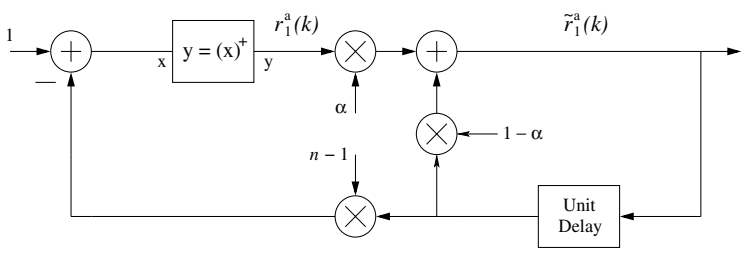

Fig. 3. Low-pass filter model of fair rate calculation.

receives a fair rate from one of the congested downstream nodes. Therefore, $\forall k, \ell_{1}(k)=1$, as there is no congested node on downstream of node 1 . Hence, from (3) we have

$$
\forall k, r_{1}^{a}(k)=\min \left\{1-r_{1}^{f}(k), \ell_{1}(k)=1\right\}=1-r_{1}^{f}(k) .
$$

As $\forall i, u_{i}(k)=r_{i}^{a}(k)+r_{i}^{f}(k)$, it follows from (7) that $\forall k$, $u_{1}(k)=1$. Therefore, $\tilde{u}_{1}(k)$ can always be obtained from (4). Note that $\tilde{u}_{1}(k)$ in (4) is an increasing function of $k$ and, consequently, $\tilde{u}_{1}(k)>\tau, k \geq \hat{k}$. This means that node 1 is congested for all $k \geq \hat{k}$. As a result, node 1 calculates and advertises a fair rate to its upstream nodes in every control interval after the congestion detection.

- In control interval $k=\hat{k}+1$, as $\ell_{i}(\hat{k}+1)=0, i>1$, it follows from (3) that $r_{i}^{a}(\hat{k}+1)=0, i>1$. In other words, node $i>1$ does not add any traffic to the ring. Consequently, $r_{1}^{f}(\hat{k}+1)=0$, and using $(7), r_{1}^{a}(\hat{k}+1)=$ 1 . Therefore, according to (1), the new calculated fair rate of node 1 is equal to $\tilde{r}_{1}^{a}(\hat{k}+1)=(1-\alpha) \cdot 0+\alpha \cdot 1=\alpha$. Note that node $i>1$ is not congested in control interval $\hat{k}+1$ as $u_{i}(\hat{k}+1)=0, i>1$. Hence, node 1 is the only congested node and all upstream nodes adjust their add-rates to the fair rate of node 1 . At the end of control interval $\hat{k}+1$, node 1 advertises $\tilde{r}_{1}^{a}(\hat{k}+1)$ as the fair rate to its upstream nodes.

- In control interval $(\hat{k}+2)$, we have $\ell_{i}(\hat{k}+2)=\tilde{r}_{1}^{a}(\hat{k}+$ $1)=\alpha, i>1$. This means that each node $i>1$ can add traffic to the ring up to the rate $\alpha$. Using (3), it follows that $r_{i}^{a}(\hat{k}+2)=\min \left\{1-r_{i}^{f}(\hat{k}+2), \alpha\right\}, i>1$. For transit traffic of node 1 , which is the aggregated traffic from nodes $2,3, \ldots$, and $n$, we have $r_{1}^{f}(\hat{k}+2)=\min \{1,(n-$ $1) \cdot \alpha\}$. Hence, the add-rate of node 1 is $r_{1}^{a}(\hat{k}+2)=$ $1-r_{1}^{f}(\hat{k}+2)=\max \{0,1-(n-1) \cdot \alpha\}$. Then, node 1 calculates its new fair rate as $\tilde{r}_{1}^{a}(\hat{k}+2)=(1-\alpha) \cdot \alpha+$ $\alpha \cdot r_{1}^{a}(\hat{k}+2)$ which is advertised to its upstream nodes.

- In general, in control interval $k>\hat{k}, \ell_{i}(k)=\tilde{r}_{1}^{a}(k-1)$, $i>1, r_{1}^{f}(k)=\min \left\{1,(n-1) \cdot \tilde{r}_{1}^{a}(k-1)\right\}$, and the add-rate of node 1 is obtained as

$$
r_{1}^{a}(k)=\left(1-(n-1) \cdot \tilde{r}_{1}^{a}(k-1)\right)^{+}, k>\hat{k},
$$

where $(x)^{+}$denotes $\max \{0, x\}$. Consequently, the fair rate of node 1 for $k>\hat{k}$ can be calculated as follows:

$$
\tilde{r}_{1}^{a}(k)=(1-\alpha) \cdot \tilde{r}_{1}^{a}(k-1)+\alpha \cdot\left(1-(n-1) \cdot \tilde{r}_{1}^{a}(k-1)\right)^{+} .
$$

It can be seen that $\tilde{r}_{1}^{a}(k)$ depends on $\alpha, n$, and the previous calculated fair rate, $\tilde{r}_{1}^{a}(k-1)$. Therefore, we can model the fair rate calculation process at node 1 using the first-order 
nonlinear discrete-time filter depicted in Fig. 3, where the unit delay is equal to one control interval. The advertised fair rate of node 1 is the response of this filter to a unit step function applied at $k=\hat{k}+1$ with initial condition $\tilde{r}_{1}^{a}(\hat{k})=0$.

3) Condition for Finite Convergence Time of the Fair Rate: Consider the filter in Fig. 3 and assume that the nonlinear block is bypassed (i.e. $y=(x)^{+}$replaced by $y=x$ ). In this case, the system is a first-order linear discrete-time filter with transfer function

$$
H(z)=\frac{\alpha}{1+(\alpha \cdot n-1) \cdot z^{-1}} .
$$

This linear system is stable when its pole is within the unit circle which yields

$$
\alpha<\frac{2}{n}
$$

We have proved in [7] that the stability condition of the nonlinear filter (Fig 3) is also given by (11).

Note that node 1 is assumed to be the most congested node and all other nodes adjust their local rates based on the advertised fair rate of node 1 . To maintain this condition, the congestion threshold, $\tau$, should be set to a value greater than $1-\frac{1}{n}$ and close to 1 . In this case, the other nodes are not congested for $k>\hat{k}$ and always set their add-rates to the advertised fair rate of node 1 .

\section{B. Analysis of the Model With Link Propagation Delay}

We now consider the effect of the link propagation delay on the fair rate calculation. Up to the congestion detection, this system works like the ideal model and the congestion detection time can be obtained from (5). We define $d$ as the one-hop round-trip propagation delay. In other words, the propagation delay of each link is equal to $\frac{d}{2}$. Fig. 4 illustrates the effect of the link propagation delay on the add-rate of node 1 in a scenario with $n=3$. It presents a typical control interval $k$, at which node 1 sends a control message to its upstream nodes.

Assume that node 1 advertises $\tilde{r}_{1}^{a}(k-1)$ as the fair rate to node 2 at $t_{0}=(k-1) \cdot T$ seconds. Node 2 receives the control message at $t_{0}+\frac{d}{2}$ seconds. Upon receiving the control message, node 2 sets its rate controller to the advertised fair rate and forwards the control message to node 3 .

At $t_{0}+d$ seconds, node 1 can see the effect of traffic change of node 2 due to the advertised fair rate $\tilde{r}_{1}^{a}(k-1)$. Note that

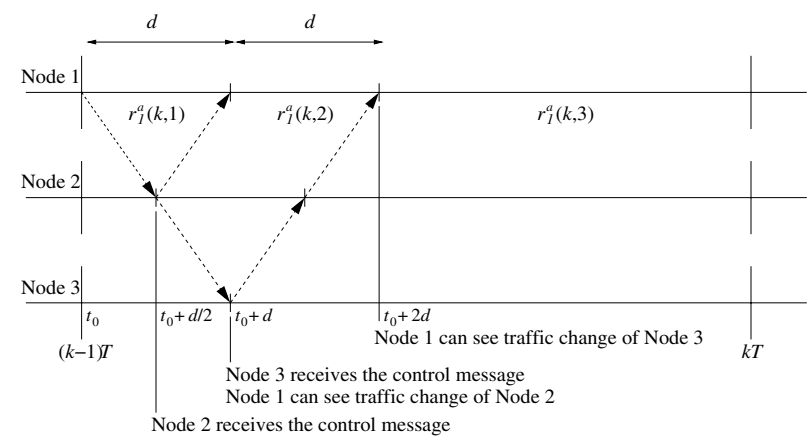

Fig. 4. Effect of propagation delay on advertising the fair rate. the forward-rate and, consequently, the add-rate of node 1 in time interval $\left(t_{0}, t_{0}+d\right]$ does not depend on the advertised fair rate $\tilde{r}_{1}^{a}(k-1)$ yet.

At $t_{0}+d$ seconds, node 3 receives the control message forwarded by node 2 . It also sets its rate controller to the advertised fair rate. Node 1 will see the effect of the rate adjustment of node 3 at $t_{0}+2 \cdot d$ seconds. In general, node 1 can see the traffic change of node $j>1$ due to the most recently advertised fair rate at $t_{0}+(j-1) \cdot d$ seconds. In order to simplify the problem, we assume that the time needed for the congested node 1 to see the traffic change of node $n$, which is the furthest node contributing to the congestion, is less than the length of control interval. That is,

$$
(n-1) \cdot d<T \text {. }
$$

Therefore, after advertising a new fair rate at the beginning of each control interval, node 1 can see the effect of the traffic change of all nodes contributing to the congestion in the same control interval. Hence, it can make a more solid estimation of the true fair rate. As node 1 gradually sees the effect of traffic changes of its upstream nodes, its add-rate changes during a control interval. In this case, the average add-rate of node 1 during a control interval should be measured in order to calculate the fair rate. In [7] we show that the average addrate of node 1 in control interval $k>\hat{k}$ is calculated as

$$
r_{1}^{a}(k)=\left(1-(n-1) \cdot \frac{d}{T}\right) \cdot r_{1}^{a}(k, n)+\frac{d}{T} \cdot \sum_{j=1}^{n-1} r_{1}^{a}(k, j),
$$

where $r_{1}^{a}(k, j)=\left(1-(n-j) \cdot \tilde{r}_{1}^{a}(k-2)-(j-1) \cdot \tilde{r}_{1}^{a}(k-1)\right)^{+}$, $1 \leq j \leq n$.

The calculated fair rate of node 1 in control interval $k$, $\tilde{r}_{1}^{a}(k)$, is obtained by substituting (13) in (1). One can see that $\tilde{r}_{1}^{a}(k), k>\hat{k}$, depends on $\alpha, n, \tilde{r}_{1}^{a}(k-2)$, and $\tilde{r}_{1}^{a}(k-1)$. As a result, using a method similar to the one applied to the ideal case $(d=0)$, we can model the fair rate calculation at node 1 by a second-order nonlinear discrete-time filter.

We implemented this analytical model in MATLAB to analyze the effect of different parameters on the calculated fair rate. We observed similar properties in the model with the propagation delay and in its corresponding linear system, which is obtained by bypassing all nonlinear blocks in (13). For instance, it seems that the stability domain of the secondorder nonlinear system (with respect to $\alpha$ for a given $\mathrm{n}$ ) is similar to its corresponding linear system. In [7], we have proved that the corresponding linear system of the model with the propagation delay is stable for a wider range of $\alpha$ compared to the corresponding linear system of the model without the propagation delay. We have also shown that the maximum range of $\alpha$ with respect to which the corresponding linear system of the model with the propagation delay remains stable, is achieved when $(n-1) \cdot d=0.5 \cdot T$.

\section{NUMERICAL RESUlTS AND ANALYSIS}

This section presents the analytical and the simulation results. The simulation model is implemented in OPNET. We consider 100Mbps links, 512 bits packet size, and $T=1 \mathrm{msec}$. 


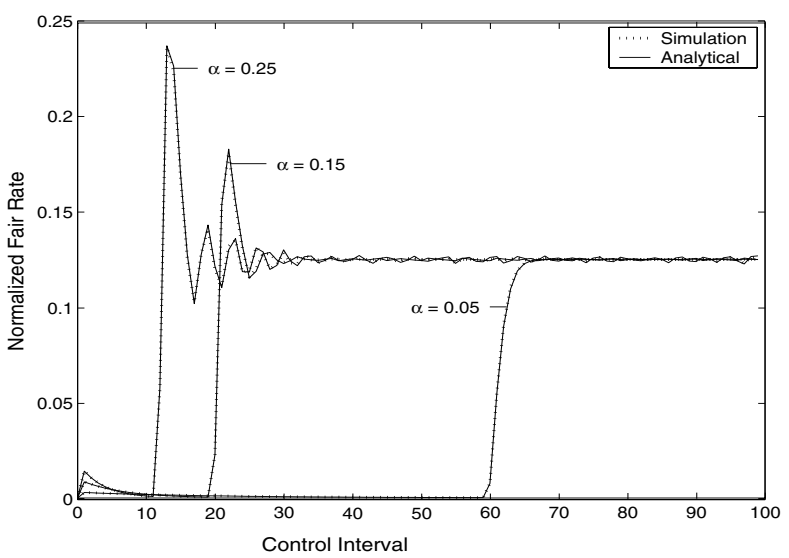

Fig. 5. Normalized calculated fair rate for $n=8, \tau=0.95$, and $d=0.1 \cdot T$.

\section{A. Effect of $\alpha$ on Fair Rate Oscillations}

The oscillations of the fair rate result from an overestimation or underestimation of the true fair rate. As the oscillations contribute to the convergence time, they are part of the performance of the fairness algorithm. Fig. 5 presents both analytical and simulation results of the calculated fair rate in a scenario with $n=8, d=0.1 \cdot T, \tau=0.95$, for $\alpha=0.05$, 0.15 , and 0.25 . One can first note that analytical and simulation results are very close. In fact, the maximum relative error in all cases is less than $2 \%$. Our analytical model is a very accurate approximation of the real system and may be used to evaluate the performance of the RPR fairness algorithm. In Fig. 5, congestion is detected at control intervals 59, 19, and 11 for $\alpha=0.05,0.15$, and 0.25 , respectively. One can see that as $\alpha$ increases, the congestion detection time decreases. However, the calculated fair rate converges to the true fair rate with a larger number of oscillations. The reason is that when $\alpha$ is large, the latest measured data has more weight in the calculation of the next fair rate, according to (1), and the true fair rate may not be estimated accurately. Therefore, the fair rate may be overestimated, then underestimated, and so on.

\section{B. Effect of Propagation Delay on Fair Rate Oscillations}

Fig. 6 illustrates the effect of the propagation delay on the fair rate calculation for $n=4$ and $\alpha=0.5(\hat{k}=5)$. Both analytical and simulation results are shown. As delay increases, the congested node sees the effect of traffic changes due to the last advertised fair rate in a smaller portion of the control interval which affects the value of the new calculated fair rate. For example for $d=0.2 \cdot T$, the first overshoot of the calculated fair rate occurs with one control interval delay comparing with the other two cases due to the propagation delay. The maximum relative error of the simulation results in all cases is less than $1.5 \%$.

\section{Effect of Propagation Delay on Stability of Fair Rate}

We define $\alpha_{n}^{*}(d)$ as the supremum value of $\alpha$ such that $\forall \alpha<\alpha_{n}^{*}(d)$, the calculated fair rate does converge to $\frac{1}{n}$ in an $n$-node scenario with $d$ one-hop round-trip propagation delay.

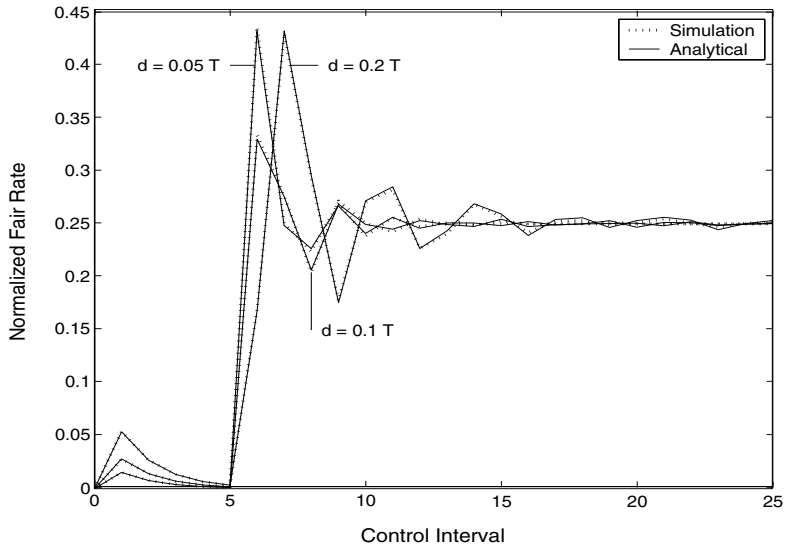

Fig. 6. Normalized calculated fair rate for $n=4, \tau=0.95$, and $\alpha=0.5$.

In the ideal model $(d=0)$, (i) the calculated fair rate in control interval $k$ depends on the calculated fair rate in control interval $k-1$ according to (9); and (ii) $\alpha_{n}^{*}(d=0)=\frac{2}{n}$ (See (11)). In the realistic model $(d>0)$, the calculated fair rate in control interval $k$ depends on the fair rate calculated in the control intervals $k-1$ and $k-2$ (See (13)). As it was mentioned in Section III-B, we observed through analytical results that that the system with delay is stable in a wider range of $\alpha$ compared to the system without the propagation delay. Therefore, $\alpha_{n}^{*}(d>0)>\alpha_{n}^{*}(d=0)$. Fig. 7 shows the analytical results of $\alpha_{n}^{*}(d)$ versus the normalized propagation delay $\left(\frac{d}{T}\right)$ for different number of nodes, $n$. It can be seen in Fig. 7 that $\alpha_{n}^{*}(d>0)>\frac{2}{n}$. As $d \rightarrow 0$, the model behaves similar to the ideal model $(d=0)$ and $\alpha_{n}^{*}(d) \rightarrow \frac{2}{n}$. In [7], we have proved that the corresponding linear system of the model with the propagation delay exhibits a unique maximum value of $\alpha_{n}^{*}(d)$ for $\frac{d}{T}=\frac{1}{2(n-1)}$. This can be observed for the actual model in Fig. 7 as well where for a given $n$, the maximum value of $\alpha_{n}^{*}(d)$ is achieved approximately for $\frac{d}{T}=\frac{1}{2(n-1)}$.

\section{Effect of $\alpha$ on Fair Rate Convergence Time}

We define $k_{c, n}$ as the convergence time of the system with $n$ nodes, i.e., $k_{c, n}=\min \left\{k:\left|r_{i}^{a}(k)-\frac{1}{n}\right| \leq \frac{\varepsilon}{n}, i=1,2, \cdots, n\right\}$,

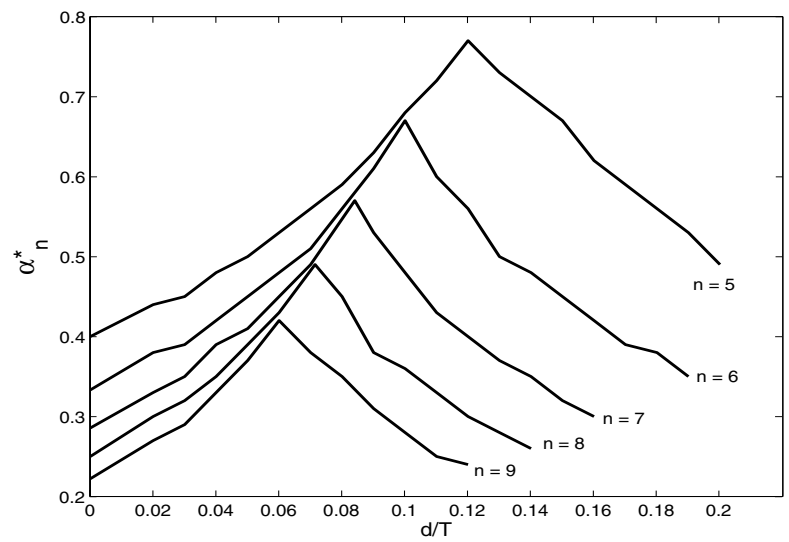

Fig. 7. Variation of $\alpha_{n}^{*}$ due to the propagation delay changes. 


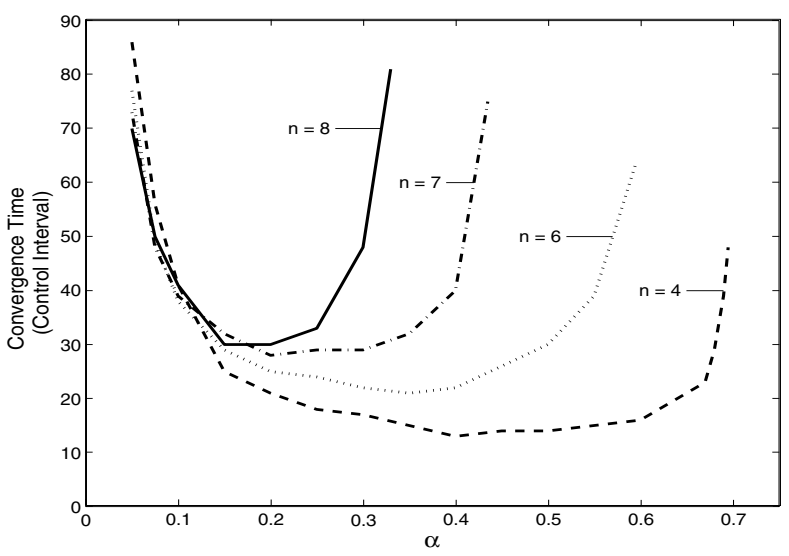

Fig. 8. Convergence time vs. $\alpha$ with $\varepsilon=0.01$ and $d=0.1 \cdot T$.

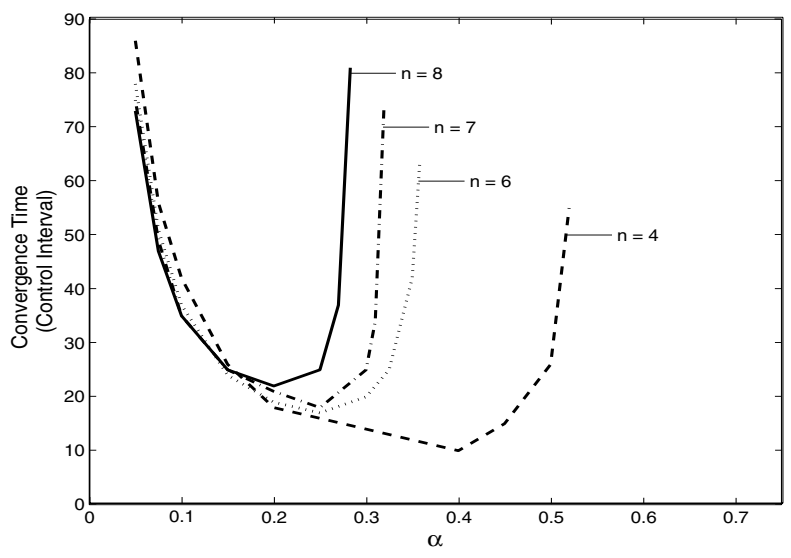

Fig. 9. Convergence time vs. $\alpha$ with $\varepsilon=0.01$ and $d=0.02 \cdot T$.

for a given $\varepsilon, 0<\varepsilon<1$. Fig. 8 shows the analytical results of the convergence time for different $n$ versus $\alpha$ for $d=0.1 \cdot T$. The convergence time consists of two components: (i) congestion detection time; and (ii) search-time which is defined as the time between the congestion detection and convergence of the fair rate when nodes search for the true fair rate. The congestion detection time is independent of the number of nodes contributing to the congestion, and is a decreasing function of $\alpha$ for a fixed $\tau$ according to (5). As $\alpha$ increases, the congestion detection time decreases but on the other hand the calculated fair rate experiences more oscillations and, hence the search-time increases.

It is noticeable in Fig. 8 that for a fixed $n$, when $\alpha$ is close to 0 , the convergence time is a decreasing function of $\alpha$. The reason is that when $\alpha$ is close to 0 , the detection time (which is a decreasing function of $\alpha$ ) is the dominant part in the convergence time.

As $\alpha$ increases, the detection time drops while the searchtime rises. Therefore, the convergence time does not change significantly and remains in the same range in some extent. For example, one can see in Fig. 8 that the convergence time for $n=4$ does not change significantly when $0.2<\alpha<0.6$. However, when $\alpha \rightarrow \alpha_{n}^{*}(0.1 \cdot T)$ (which is for example around
0.7 for $n=4$ in Fig. 8), the search-time is the main source of delay in the convergence time and its drastic increase.

We also observe that when $\alpha$ is relatively small (e.g., $\alpha<$ $0.05)$, for $n_{1}>n_{2}$, we have $k_{c, n_{1}}<k_{c, n_{2}}$. The reason is that for small values of $\alpha$, the calculated fair rate does not oscillate and instead increasingly converges to the true fair rate (this is shown in Fig. 5 for $\alpha=0.05$ ). For the true fair rates we have $\frac{1}{n_{1}}<\frac{1}{n_{2}}$, hence $k_{c, n_{1}}<k_{c, n_{2}}$.

It can also be seen that as $\alpha$ increases and the estimated fair rate oscillates, the system with more nodes faces more delay in converging to the true fair rate. The reason is that for $n_{1}>n_{2}$, we have $\alpha_{n_{1}}^{*}(d)<\alpha_{n_{2}}^{*}(d)$ and when $\alpha$ is within the stable domain of both systems, it is closer to $\alpha_{n_{1}}^{*}(d)$ than $\alpha_{n_{2}}^{*}(d)$. In this case, we can conclude that convergence time of the system with more node is larger. In addition, any change in the propagation delay can increase or decrease $\alpha_{n}^{*}(d)$ according to Fig. 7. For example, we observe in Fig. 7 that, by decreasing the link propagation delay from $d=0.1 \cdot T$ to $d=0.02 \cdot T, \alpha_{n}^{*}(d)$ decreases for $n=6,7$, and 8 . Fig. 9 depicts the analytical results of the convergence time for different $n$ with $d=0.02$. $T$. By comparing graphs in Figs. 8 and 9, it can be seen that $\alpha_{n}^{*}(0.02 \cdot T)<\alpha_{n}^{*}(0.1 \cdot T)$. From results of Figs. 8 and 9 , we heuristically conclude that for a system with the propagation delay, the convergence time of the fair rate is relatively small for $\alpha$ such that $\frac{1}{n}<\alpha<\frac{2}{n}$.

\section{CONCLUSION}

We developed an analytical model for fair rate calculation in the standard RPR fairness algorithm in the parking lot scenario. Our approach is to model the fair rate calculation process as a nonlinear discrete-time low-pass filter. Simulation results confirm the model accuracy. This model can be used to evaluate performance of the RPR fairness algorithm in terms of stability and convergence time. Using this model, we studied the effect of various system parameters on stability and convergence of the calculated fair rate. Finally, we concluded that by choosing the low-pass filter coefficient $\alpha$ so that $\frac{1}{n}<\alpha<\frac{2}{n}$, the convergence time of the fair rate in the parking lot scenario is within its minimum range.

\section{REFERENCES}

[1] M. Karol, and R. Gitlin, "High performance optical, local and metropolitan area networks: Enhancements of FDDI and IEEE 802.6 DQDB," IEEE JSAC, vol. 8, no. 8, pp. 1439-1449, October 1990.

[2] F. Davik, M. Yilmaz, S. Gjessing, and N. Uzun, "IEEE 802.17 Resilient Packet Ring Tutorial," IEEE Communication Magazine, vol. 42, no. 3, pp. 112-118, March 2004.

[3] I. Cidon, L. Georgiadis, R. Guerin, and Y.Shavitt, "Improved fairness algorithms for rings with spatial reuse," IEEE/ACM Transactions on Networking, vol. 5, no. 2, pp. 190-204, April 1997.

[4] R. Simha, and Y. Ofek "A Starvation-free Access Protocol for Fullduplex Buffer Insertion Ring Local Area Networks," Journal of Computer Networks and ISDN Systems, vol. 21, 1991.

[5] V. Gambiroza, P. Yuan, L. Balzano, Y. Liu, S. Sheafor, and E. Knightly, "Design, analysis, and implementation of DVSR: A fair, high performance protocol for packet rings," IEEE/ACM Transactions on Networking, vol. 12, no. 1, pp. 85-102, February 2004.

[6] IEEE, IEEE Standard 802.17: Resilient Packet Ring (Draft Version 3.1), http://ieee802.org/17, February 2004.

[7] A. Shokrani, I. Lambadaris, J. Talim, "An Analytical Model for Fair Rate Calculation in Resilient Packet Ring," Carleton University Technocal Report, SCE-04-16, August 2004. 